\title{
EL IDEAL BRASILEÑO FRENTE A LOS CASOS DE DISCRIMINACIÓN POR ORIENTACIÓN SEXUAL EN EL ÁMBITO DE LA ENSEÑANZA NACIONAL
}

\author{
THE BRAZILIAN IDEAL AGAINST CASES OF DISCRIMINATION BASED ON SEXUAL \\ ORIENTATION IN THE FIELD OF NATIONAL EDUCATION
}

\author{
Douglas Verbicaro Soares \\ Universidade Federal de Roraima, Boa Vista, RR, Brasil. Doutor em Direito \\ http://orcid.org/0000-0002-9242-9124
}

DOI: https://doi.org/10.46550/amormundi.v1i2.20

Recebido em: 12.09.2020

Aceito em: 28.10.2020

\begin{abstract}
Resumen: La investigación trata sobre la incidencia de prácticas discriminatorias motivadas por la no aceptación de la diversidad sexual en Brasil y, también, sobre las dificultades en la implementación de medias en favor de la sensibilización en cuestiones de educación para los Derechos Humanos. De esa forma, ha sido empleado el método cualitativo para el estudio, a través de materiales bibliográficos, con el objetivo de generar una discusión social en el país sobre los obstáculos existentes para la efectiva integración de grupos en situación de vulnerabilidad social. Si ha concluido por la incidencia de acciones discriminatoria que implementa la predominancia de la heterosexualidad en relación a las demás formas de expresión de la sexualidad humana.
\end{abstract}

Palabras- llave: Sexualidad. Desafíos. Derechos Humanos. Conflictos.

Abstract: The research deals with the impact of discriminatory practices motivated by The Non-Acceptance of Sexual Diversity in Brazil and also on the difficulties in implementing means for raising awareness of Human Rights education issues. In this way, the qualitative method for study, through bibliographic materials, has been used with the aim of generating a social discussion in the country on the existing obstacles to the effective integration of groups in situations of social vulnerability. If you have concluded by the incidence of discriminatory actions that implements the predominance of heterosexuality in relation to the other forms of expression of human sexuality.

Keywords: Sexuality. Challenges. Human Rights. Conflicts.

\section{Introducción}

Esta investigación se basó en la investigación bibliográfica, con énfasis multidisciplinario
en el tema de la educación inclusiva para las personas homosexuales dentro de la sociedad brasileńa, visibilizando prácticas discriminatorias y prejuiciosas contra los LGBTs (lesbianas, gais, bisexuales y transexuales) en Brasil. 
El desarrollo del estudio comenzará con un enfoque histórico de los informes de comportamientos estigmatizantes para estas víctimas, haciendo hincapié en la viabilidad de combatir los obstáculos existentes que refuerzan el trato desigual entre todos los ciudadanos del país. Los informes visualizados en la investigación convirtieron a homosexuales en personajes de segunda categoría, es decir, a quienes se les negó la participación social, política, económica y religiosa, por ejemplo. Así, sin olvidar que estas personas fueron olvidadas, durante mucho tiempo, por el Estado brasileño, a través de pocas e insuficientes políticas públicas que les permitieron superar las situaciones de vulnerabilidades a las que se enfrentan.

Por esta razón, el estudio pretende gestionar el tema de la orientación homosexual en el ámbito de la educación, destacando que la orientación homosexual representa sólo una de las manifestaciones de las sexualidades existentes, sin razón, por lo tanto, para la incidencia de acciones que generan la exclusión social de las personas LGBTs, especialmente los homosexuales que representan el objeto de la investigación.

El artículo se dividió en dos secciones, la primera se centró en informes de prácticas discriminatorias y la segunda explicando la educación como ideal para la integración. Seguido de las consideraciones y referencias finales. También para la investigación se casaron algunas preguntas: ¿Hay informes en Brasil que indiquen la incidencia de prácticas discriminatorias contra las personas homosexuales en el contexto de la enseñanza? ¿Puede la educación servir como instrumento para la conciencia social en el país sobre cuestiones de diversidad sexual?

\section{Los relatos de acoso y discriminación por orientación sexual en Brasil}

Para nuestro estudio no resta duda que el instrumento de la educación (VERBICARO SOARES, 2012, p. 56) representa un factor predominante de los cambios necesarios en la sociedad de Brasil (RODRIGUEZ, 2001, p. 134-5), pues los grupos víctimas de prejuicio y discriminación como han sido presentados en este estudio, buscarán sus espacios en las profesiones intelectuales y administrativas, retirando importantes modificaciones en la división del trabajo, pero que todavía se encuentran aisladas, impedidas de ocupar los cargos de autoridad y de mayor responsabilidad como ocurre en los sectores de la economía, finanzas y política.

De este modo nos pareció oportuno el comentario de Maquieira Dángelo:

La preocupación por falta de un trasvase eficaz entre las investigaciones llevadas a cabo desde las diferentes disciplinas de ciencias sociales utilizando el género como instrumento de análisis y los libros de texto escolares en la enseñanza secundaria Obligatoria y el bachillerato nos llevó a la necesidad de denunciar, una vez más, el estado de la cuestión e incidir, si era posible, en un cambio inaplazable. A partir de esa primera reflexión planteamos un proyecto con unos objetivos que pretendían no sólo investigar donde estaba el problema y qué profundidad alcanzaba sino también contactar con profesoras y profesores preocupados por educar en igualdad, con inquietud ante la falta de instrumentos y, sobre todo, aislados en sus Centros, sin contacto con grupos de investigación con los que intercambiar preocupaciones y experiencias (DÁNGELO, 2005, p. 433).

Eses instrumentos sirven para evitar la repetición de casos de acoso escolar por motivos de orientación sexual, en nuestro caso, la homosexual, como ha sido el caso del rechazo de la matricula en una institución de enseñanza de Brasil, debido a la manifestación afeminada de niños o en los casos más extremos de expulsión de un menor por lo mismo motivo alegado supra, 
como se ha escuchado que se justificaría por: se evitar que un futuro podrido contaminara el resto de los alumnos (MOTT, 1997, p. 19-20).

Otro relato en Brasil ha sido en Río de Janeiro en 1993, cuando dos alumnas lesbianas de la Escuela Miguel Couto, en el barrio carioca de la Tijuca, han tenido sus matrículas anuladas y que también han sido violentadas por centenas de estudiantes (tanto de la escuela como de otro instituto cercano), cuando las dos han decidido asumir públicamente su orientación sexual (MOTT, 1997, p. 19-20). Del mismo modo en que el estudiante Cláudio José Pena, ha sido expulsado de la residencia universitaria en la cual vivía, bajo la acusación de practicar actos homosexuales en su habitación en la provincia del Rio Grande do Norte.

También en la Escuela Superior de Diplomacia (Instituto Rio Branco), se han detectado registros de episodios discriminatorios cuanto el acceso a la educación. Citaremos en caso de Carlos Loureiro Carvalho, que había sido expulsado del Instituto por sospechas de ser homosexual. También fue en el período de la dictadura militar en Brasil que Vítor Irrigaría, en su expediente en el Itamaraty, según su laudo médico, firmado por un psiquiatra (André Santiago Lima, que en la época era militar, más específicamente teniente de la Marina de Brasil, contaba en su registro que era portador de una estructura psicológica homosexual, por lo menos latente). Hecho que más tarde culminó con la expulsión del curso al que realizaba por su orientación sexual homosexual (MOTT, 1997, p. 21-2).

Hechos que representan claramente ciertas ideas discriminatorias hacia a las personas que son diferentes en un modelo fuertemente moldado el roles de género y en estereotipos de masculinidades y feminidades. Un otro caso mencionado ha ocurrido en 1993, en la Escuela Zulmira Ribeira Torres de Salvador, en la Provincia de Bahia en Brasil, donde un niño de 13 años en la época, ha tenido que cambiar de aula para escapar de los continuos acosos físicos y psicológicos que sufría por parte de sus compañeros de aula (MOTT, 1997, p. 19-20).

Estos hechos nos remite a otro, de proyección internacional, ocurrido en finales de 2013, cuando se ha premiado la cantante Lady Gaga en la Universidad de Harvard de EE.UU., por el labor realizado por su fundación: "born this way" (nacido de este modo), en el combate al acoso escolar o el denominado "bullying" (ABC.ES, 2013). La autora, por su destaque internacional, ha comentado en la ocasión que había sido víctima del mismo acoso cuando era niña, por ser gorda por sus colegas. Esas declaraciones han generado toda una discusión social sobre ese problema, no solamente en Norte América, pero en todo el mundo. La visibilidad del problema sirve para combatirlo y a motivar a las personas que lo sufren a enfrentarlo.

La citación de la artista tiene que ver con un episodio trágico de 2011, cuando un joven norte americano Jamey Rodemeyer, un seguidor de Lady Gaga, se suicidó debido al fuerte acoso que sufría por ser homosexual en su instituto. Ha sido notorio mundialmente este caso, que la cantante, se empeñó en concienciar la sociedad internacionalmente sobre el problema del bullying escolar y lo grave que puede ser, los riesgos que representan. Tanto es cierto, que después del ocurrido, la cantante por medio de su fundación, ha cobrado incluso del presidente Barack Obama una movilización más serie del Gobierno de los EE.UU en tratar de esa problemática (ANTENA3, 2014).

Para Rodrigues:

Desde que el individuo es sometido al proceso de socialización primaria, se intenta adaptar esta persona a las normas sociales, de disciplinar para la 
convivencia en sociedad, por medios de estancias de controles informales. Este proceso que comienza en la familia, pasa por la escuela, la religión, la profesión, el trabajo, convirtiendo estos agentes sociales en estancias de controles informales (RODRIGUEZ, 2001, p. 134-5).

El texto explicita que las primeras relaciones son fundamentales para la formación de un individuo, serían a través de ellas que tomamos conocimiento de nuestras primeras referencias de vida y trato con los demás en sociedad. Se no existe una buena educación, o que esté ausente alguna disciplina necesaria para la evolución histórica de una persona en los medios de controle informal, las chances de desvío serán muy grandes para la no adecuación en sociedad de esa persona (VERBICARO SOARES, 2012, p. 56).

\section{La educación como ideal para la integración}

\section{De acuerdo con Dennis Verbicaro:}

La educación debe de ser una forma de poner en prueba y de añadir, de desarrollar los sentidos para la apreciación de las distintas artes. De estimular la curiosidad para buscar secretos de la naturaleza y de la historia. Deberá de ser sorprendente, divertida, cautivadora. Nuestro universo pequeño se rompe y sentimos el crecimiento. Derrumbamos las fronteras de nuestro interior y nos ponemos dentro de un grupo de seres fundamentalmente iguales a nosotros. De ahí surge la primera enseñanza sobre la democracia moral, que será aquella que nos llevará a trascender el grupo étnico, la Nación y al Estado, y llevará en consideración los intereses de los que son iguales (VERBICARO 2009, p. 16).

Se reconoce el papel de destaque que asume el instrumento de enseñanza para las personas puedan desarrollar sus proyectos de conquista de la ciudadanía. De modo contrario a ese posicionamiento, Brasil, durante un largo período de tiempo, sigue un camino preocupante en relación al problema de la educación. Se puede argüir que la temática de la educación acaba siendo recordada solamente en el discurso político, y que después el tema acaba siendo olvidado o tratado con desprecio por los propios gobernantes del país.

Al analizar los comentarios supra mencionados, podemos aducir que la educación es un instrumento que permite un desarrollo de los sentidos, se plasma en la descubierta por las cosas, tanto por la naturaleza, como también por la historia de la humanidad, es un conjunto de características que posibilita sobretodo romper de las fronteras más internas y catapultar a la descubierta por la diversidad, del diverso y ampliar la visión sobre las cosas, como por ejemplo puede ser: el aprendizaje de un cálculo numérico, o en la lectura de un cuento, también está en los aprendizajes de una actitud solidaria de auxilio a las personas más mayores, o a través del respeto al que es diferente, como ocurre con los temas de raza, sexualidad, violencia de género, origen, cultura, religión, política, etcétera (VERBICARO SOARES, 2012, p. 58).

La educación, por lo tanto, representa la instrumentación del camino para la manutención de la armonía social, es decir: en la convivencia con el mutuo respeto y de trato igualitario y justo entre las personas. Ésta es la base para la protección y la real efectividad de los derechos, sean instrumentalizados de modo internacionalizado o de modo más positivo, dentro de los Ordenamientos Jurídicos de los Estados, constituidos dentro de garantías fundamentales.

Con relación a estos argumentos estamos de acuerdo con Soriano Rubio, que defiende la necesidad de no solamente evidenciar la homosexualidad como existente en distintas sociedades 
por el mundo y que esa orientación sexual deberá ser entendida como saludable. Para los cambios necesarios es preciso enfocar en la educación en tres pilares: 1) El análisis de lo que sería la homosexualidad, combatiendo la desinformación general sobre ella, realizar la diferencia entre orientación y conducta sexual y reiterar la propia multidiversidad que el tema engloba; 2 ) entender que cualquier persona podrá ser homosexual, sin la incidencia de padrones estereotipados que influían en la homosexualidad de una persona: que aquellos que estén empezando a dar cuenta de sus deseos homosexuales en lugar de la confusión y negación no puede ser, estaré equivocado o a mí no me puede estar pasando, se puedan llegar a decir: es posible o también me puede suceder a mí. De este modo, permitiendo que las personas que no son homosexuales puedan tomar conciencia de la posibilidad de que ésta se dé en otras personas similares, como a un amigo, un familiar por ejemplo; 3) analizar las actitudes sociales y los problemas vividos por algunas personas su propia sexualidad: conocer estos temas que conllevan la aceptación como homosexual, tanto para las personas con esa orientación sexual, como para los que no la tienen, conociendo de un modo más amplio por la sociedad lo que implica aceptarse como homosexual y asumir su sexualidad (RUBIO, 1999, p. 187-9):

Los educadores deberían reflexionar y tomar consciencia de la necesidad de trabajar el tema de la sexualidad en las aulas, que a menudo bien por las dificultades, bien por la falta de información o bien por las actitudes personales y sociales ha sido el gran olvidado y ocultado por todos.

De este modo, las personas, por más diversas que puedan ser en su características, tienen una única esencia en común, que todos somos humanos. Esa idea se asemeja a la defendida por Norberto Bobbio en relación a la definición de los derechos del hombre, que serían los derechos que el reconocimiento de los mismos incide en la condición necesaria para el desarrollo de la persona humana, u para el desarrollo de la civilización (BOBBIO, 1992, p. 17).

Nos gustaría complementar esa idea con una citación que no tiene un carácter científico, pero que no deja de aportarnos una cierta belleza artística para comprehender la grandiosidad de ser humanos. En la obra "Contacto" del norteamericano Carl Sagan, nos presenta sus ideas sobre los méritos de los humanos, que han sido capaces de desarrollar artísticamente:

La música, la benevolencia, los sueńos. Los humanos poseen una habilidad especial para los sueños. Anoche exploramos el interior de cada uno de vosotros, y encontramos muchas cosas: sentimientos, recuerdos, conductas adquiridas, rasgos de locura, sueños, amores. El amor es muy importante. Sin lugar a dudas, constituyen una interesante mezcla (SAGAN, 1986, p. 327).

Para la finalización de éste apartado, reiteramos nuestra convicción de que la educación (VERBICARO 2009, p. 16) es fundamental para concienciar y permitir la aceptación de la orientación sexual en la sociedad brasileña, así como en otras sociedades internacionales. Una vez que se naturalice el valor positivo que se tiene de ser homosexual, y se logre una instrucción basada en valores de igualdad, de podrá combatir los estereotipos, los mitos, las fobias en relación a ese tema.

En este estudio científico reiteramos la exigencia de si investir en medias de prevenciónprotección en el ámbito de la sexualidad humana, una vez que los daños generados por las situaciones de victimización, vulneración de derechos y tratos desiguales pueden generar toda una problemática, tanto para la persona que sufre la situación de desigualdad, así como para la sociedad en general, es decir para el sujeto pasivo (lo que recibe la acción negativa) pude generar 
situaciones de actitud temerosa, ansiedad silenciosa, apatía, infelicidad, tristeza, autoestima baja, falta o incremento del apetito, sobresaltos continuos es sus actos, actitudes de depresión, intentos extremos de suicidios y consumación del mismo, consumo evasivo de tóxicos - abuso de alcohol, drogas y psico-fármacos (VERBICARO SOARES, 2012, p. 58).

Estos hechos pueden ser considerados como manifestaciones con resultados fatales para las víctimas y para su salud física y mental, pudendo incluso a agraves las patologías de las personas que sufren con la violencia (lesiones diversas, heridas, condiciones crónicas de salud - dolor crónico, síndrome del intestino irritable e otros trastornos gastrointestinales, quejas somáticas, repercusiones en la salud sexual y reproductiva - sangrados, pérdida del apetito sexual), sea cual sea su origen. Las repercusiones en la esfera de la salud social pueden ser: aislamiento, pérdida del empleo, absentismo laboral, disminución del número de días de vida saludable (VERBICARO SOARES, 2012, p. 58).

Las consecuencias en la vida de otras personas cercanas a la víctima, como por ejemplo los niños, que también pueden empezar a desarrollar comportamientos temerosos, de miedo, posturas de ignorancia, de pasotismo, de no aceptación, sentimientos de vergüenza, actitudes de silencio y de encubrimiento, afectando de ese modo, la salud de los hijos, representando un verdadero riesgo para ellos, alterando su desarrollo integral.

Las actitudes de discriminación también son perjudiciales para la sociedad en general, pues el Estado se ve obligado a buscar, investir en instrumentos para cohibir esas conductas, teniendo que hacer más inversiones en políticas sociales, incremento económicos para las fuerzas de seguridad, a la sanidad pública, todo generando un alto gasto económico para el trato de todas las personas envueltas con esas problemáticas. Por este motivo, es válido incentivar la prevención de la violencia utilizando la educación como medida libertadora social (VERBICARO SOARES, 2012, p. 58).

La opinión de Melo nos revela que:

Distintos estudios tienen mostrado que sin un proceso masivo de educación, en todas las esferas de la sociedad, es prácticamente imposible asegurar una igualdad legal y social entre diferenciados grupos socio-sexuales en cualquier sociedad, pues nadie nace homofóbico, tampoco homofílico, siendo nuestras creencias, valores y actitudes resultantes de un proceso de aprendizaje social. En cuanto los hombres, pertenecientes al sexo masculino fueren estimulados por sus padres, por la escuela, por la iglesia y por los medios de comunicación de masa a considerar homosexuales y transgéneros seres humanos inferiores y ciudadanos de segunda categoría, una ley que puna la homofobia, por sí sólo, no será capaz de parar la violencia contra gais, lesbianas y transgéneros (MELLO, 2009, p. 167).

En contrapartida, la situación brasileña referente al lema de la educación en el ámbito público, que debería pertenecer a todos los ciudadanos se encuentra en un cuadro lamentable:

Se hace fundamental una dedicación más eficiente del Gobierno de Brasil a afrontar los altos índices de repetidores en los cursos, con un resultado insuficiente, principalmente en los niveles iniciales, lo que contribuye para el aumento del abandono escolar por parte de los niños brasileños; Del mismo modo, es necesario investir en la aplicación de la enseñanza fundamental, donde el limitado número de vagas todavía no es suficiente para la cantidad de ciudadanos que necesitan seguir sus estudios.

La ausencia o precariedad de los cursos disponibles cuyas plazas son limitas, impiden que 
muchos alumnos sigan sus estudios. Hechos que dificultan sobremanera la calidad de la enseñanza ofertada por el Gobierno, que peca en la calidad y que parece ser ineficaz a la formación de sus ciudadanos, lo que implica en el aumento de las dificultades para que los jóvenes brasileños puedan insertarse en el mercado laboral.

Por esta razón, encontramos ciertos esfuerzos del Gobierno, como será visto a seguir, en la creación de instrumentos para permitir la mejora de la enseñanza en el país, entre los temas discutidos, parece que el tema de la orientación sexual homosexual ya se muestra presente, con la creación de políticas públicas específicas, materializadas en planes de acción, para mejorar la calidad de la educación e introducir el ideario de la educación para la integración de la diversidad sexual. Entre algunas medidas sugeridas estarían: la formación de los profesionales del sector, mejorar la situación laborar de los profesores, capacitarlos, aumento de saldos, oferta de materiales didácticos de calidad, mejorar la situación estructural de las escuelas públicas, construcción de nuevas escuelas, implementar un sistema de transparencia para la distribución de los recursos destinados a la educación.

Con base en las ideas presentadas, se comprenderá la educación como el mecanismo político de los cambios, la responsable por el desarrollo. Un buen proyecto de ciudadanía consiste en la realización de sustituciones de algunos términos, es decir, educar es formar y no adiestrar, es crear un sujeto social y no un receptor pasivo del saber, es aprender a conquistar y a no recibir una concesión de la ciudadanía. El gobierno debe permitir que sus ciudadanos puedan desarrollar sus calidades individuales, así ter su dignidad alcanzada.

En este sentido, nuestro trabajo reconoce la importancia de la educación un mecanismo para que los ciudadanos brasileńos puedan conocer sus derechos y deberes en la sociedad en que viven. En este sentido, se puede afirmar que el conocimiento de las realidades de una sociedad puede generar el combate a las diferencias y problemas sociales existentes, sirviendo para la prevención y la eliminación de la exclusión, sea en el ámbito social, político o económico (MELLO, 2009, p. 167).

Tanto es cierto, que en la entrega del premio Internacional Nobel de la Paz de 2014, se ha dado un destaque mayor al tema de los Derechos Humanos, y el reconocimiento de la educación para cambios en las realidades más adversas. El caso de los vencedores, Malala Yousafzzay y de Kailash Satyarthi que, se destacaron por incrementar la lucha por los derechos de los niños, enfocando en su caso la educación como instrumento de liberación contra la represión (NOBELPRIZE, 2014).

En 2013, un año antes de la entrega del premio Nobel supra mencionado, la UNICEF lanza el Informe: "Ocultos a plena luz", haciendo referencia a un análisis estadístico de la situación de la violencia contra nińos por el mundo, en este sentido se presentó el trabajo:

La protección de los niños contra toda forma de violencia es un derecho fundamental garantizado por la Convención sobre los Derechos del Niño y otros tratados y normas internacionales de derechos humanos. Sin embargo, e independientemente de las circunstancias económicas, sociales, culturales, religiosas o étnicas de los niños, la violencia aún es un componente muy real de sus vidas en todo el mundo (UNICEF, 2014).

Donde ya se defendía la importancia del tema de la protección de los menores vulnerables y de sus derechos en el mundo. 
Los esfuerzos generados se centran en la viable consolidación de presentar la educación como instrumento de cambio necesario para la construcción de nuevos mecanismos de sensibilización que posibiliten el desarrollo amplio y participativo de la sociedad en general. La instrucción para la integración de la diversidad humana y el descubrimiento de las diversas manifestaciones de la sexualidad humana, sea heterosexual, homosexual, bisexual o incluso asexual.

La búsqueda por el respeto y la construcción de una sociedad más justa e igualitaria en Brasil, así como para muchas otras que comparten extremas dificultades para la efectividad de los derechos. La deseada emancipación ciudadana participativa, el combate a las injusticias la discriminación y el prejuicio, conseguir la igualdad de trato entre las personas y en consecuencia, buscar la armonía social, no se puede esperar la buena voluntad del Estado, o soñar con una utópica idea de democracia para el respeto de derechos.

Recordaremos las palabras de Baldassarre, que al preceptuar los límites sociales de la democracia:

El proceso de desarrollo de la democracia tiende a producirse a expensas de la misma. Más exactamente, creaciones de condiciones más favorables al desarrollo de los grupos y las expectativas de que la democracia implica, produce una sobrecarga de preguntas acerca de las instituciones políticas, que con el tiempo conducirán a la irracionalidad en la toma de decisiones y rojo en el funcionamiento de las instituciones representativas que ponen en grave peligro la estabilidad de las democracias ellos mismos (BALDASSARRE et al., 1996, p., XII).

Así, reforzando la relación entre el desarrollo de la democracia a la creación de oportunidades para los grupos que la componen.

\section{Consideraciones finales}

El estudio ha reforzado la importancia de la educación como un factor predominante de los cambios necesarios en la sociedad de Brasil contra la no aceptación social en el país de la diversidad sexual. En el mismo sentido, la investigación apuntó la necesidad de retirada de los obstáculos que impiden las personas homosexuales al acceso a los mismos derechos que los demás ciudadanos, sin sufrir discriminación por tener una orientación sexual diversa de la heterosexual dominante.

De modo preocupante, Brasil está en el topo de los países más violento en el mundo para las personas LGBTs, es decir, todos los días son visibilizados en los medios de comunicación prácticas que violan la integridad física y mental de estas personas que siguen viviendo hace tiempos en situación de exclusión social. Así, debemos indagar: ¿’hasta cuando la diseminación del odio y las LGBTfobias seguirán estigmatizando esas víctimas en Brasil?

Los problemas visibilizados en la investigación demuestran la necesidad urgente de implementación de medidas reales por la sensibilización social en el país en cuestiones de enseñanza para la igualdad y el respeto a los Derechos Humanos. Además, de destacar la viabilidad de desarrollo de nuevos estudios sobre la diversidad sexual y igualdad de género para romper paradigmas y combatir las desigualdades estructurales de un Brasil históricamente en deuda con las personas LGBTs. 


\section{Referencias}

ABC.ES. Lady Gaga, la mujer del año que lucha contra el acoso escolar. Disponible en: <http://www.abc.es/estilo/gente/20131112/abci-lady-gaga-mujer-201311121002.html>. Acceso en: 11 ene. 2021.

ANTENA3. Lady Gaga exige más medidas contra ela coso escolar a los homosexuales en la Casa Blanca. Disponible en: <http://www.antena3.com/celebrities/famosos-pop/lady-gagaexige-mas-medidas-acoso-escolar-homosexuales-casa-blanca_2011120700159.html>. Acceso en: 11 ene. 2021.

BALDASSARRE, Antonio; BALDASSARRI, Mario; BONANTE, Luigi; DAHL, Robert A; D'AMOJA, Fulvio; EMERSON, Thomas I; GUSTAFSSON, Bo; LINDBLOM, Charles E; MORTILLARO, Felice; OLSON, Mancur; RODOTEAGRAVE, Stefano; ROSE, Rose. I limiti della democrazia. Seconda edizione. Bari, Itália: Editori Laterza, 1996.

BOBBIO, Norberto. A era dos direitos. 17ª Tiragem. Rio de Janeiro, Brasil: Ed. Campus, 1992.

DÁNGELO, Virginia. Democracia, Feminismo y Universidad en el Siglo XXI. Instituto Universitario de Estudios de la Mujer. Madrid, España, 2005.

MOTT, Luiz Roberto. Homofobia. Aviolçáo dos Direitos Humanos de Gays, Lésbicas \& Travestis no Brasil. Grupo Gay Da Bahia / International Gay and Lesbian Human Rights Commission. Salvador, Brasil, 1997.

MELLO, Luiz: A escola e @s filh@s de lésbicas e gays: reflexôes sobre conjugalidade e parentalidade no Brasil. In.: JUNQUEIRA, Rogério: Diversidade Sexual na Educaçáo: problematizaçóes sobre a homofobia nas escolas. Brasília, Brasil: Coleção Educação para todos, 2009.

NOBELPRIZE. Nobel Peace Prize. Disponible en: <http://www.nobelprize.org/>. Acceso en: 11 ene. 2021.

RODRIGUEZ, Laura. La Política Criminal. 1ª Edición. Madrid, España: Colex, 2001.

RUBIO, Sonia. Como se vive la homosexualidad y el lesbianismo. Salamanca, España: Amarú Ediciones, 1999.

SAGAN, Carl. Contacto. Barcelona, España: Círculo de Lectores, 1986.

UNICEF. Ocultos a plena luz. Disponible en: <http://www.unicef.org/ecuador/ocultos_a_ plena_luz.pdf>. Acceso en: 11 ene. 2021.

VERBICARO, Dennis. Consumo e cidadania: da criaçáo à expressáo da solidariedade nas relaçóes de consumo no Brasil. Trabajo de Grado. Universidad de Salamanca: Programa de Doctorado Pasado y presente de los Derechos Humanos, Salamanca, España, 2009.

VERBICARO SOARES, Douglas. Las mujeres y las personas homosexuales en las Fuerzas Armadas. Especial referencia a las FFAA Brasileñas. Trabajo fin de Máster. Programa Estudios Interdisciplinares de Género y Políticas de Igualdade. Faculdade de Direito. Universidade de Salamanca - USAL, Salamanca, España, 2012. 146 p. 